Chan Zuckerberg Initiative (CZI)

Essential Open Source Software for Science, Cycle 2

Center for Open Science (COS) Proposal:

Advancing sustainability and innovation of open manuscript services 


\section{Abstract/Proposal Summary}

Preprints (early, complete versions of manuscripts made available online before journal-organized peer review) are shifting the scholarly publishing model by accelerating open access and, potentially, open review. Making these tools interoperable with preprint infrastructure will increase the confidence of the scientific community in preprints. In addition, it will influence commercial publishing services to embrace new business models to innovate towards openness.

OSF Preprints is open-source software maintained by the Center for Open Science (COS). OSF Preprints hosts 26 community-run services, providing the ideal conditions to integrate with open review platforms such as Peer Community In (PCI), PREreview, and Hypothes. is and to assess whether a fully open model can compete with and disrupt scholarly publishing across disciplines. We integrated hypothes.is and now propose to add the other platforms.

With support from the Alfred P. Sloan Foundation, we are investigating how to improve trust in preprints with signals of credibility to improve chances that an open model can innovate scholarly publishing. Making those signals standard, interoperable, and machine-readable with validation will facilitate adoption and impact. We propose to build open-source open science badges for preprints to be interoperable with other scholarly publishing platforms.

In 2020, COS advanced its sustainability plan for the preprints infrastructure with a distributed cost model for the shared infrastructure. Six of the services primarily serve under-resourced scholarly communities in the developing world. We request the costs for maintenance of those services for 2020 to extend their opportunity to develop institutional support for sustaining their services.

\section{Work Plan}

Preprints are advancing, but not yet competing with, the traditional publishing model. Authors must still engage the largely closed, commercial publishing services to obtain the credibility and rewards they need for career advancement. To truly disrupt commercial publishing, we must increase the credibility of research shared via open services like preprints to the level of published scholarly communications. We will: 1) make available indicators for behaviors that researchers rated as credibility enhancing, 2) integrate open peer review with preprints infrastructure to address the credibility gap between preprints and journal articles, and 3) provide support for under-resourced research communities for sharing preprints through sustainable platforms. 
Add credibility signaling badges with link validation to OSF Preprints and make openly available for broader adoption

With support from the Alfred P. Sloan Foundation to assess the credibility of preprints through a survey of 3,758 researchers, COS plans to learn which features of preprints and preprint services are most important for signaling credibility and trust. Based on that survey, OSF Preprints infrastructure will soon capture metadata for Conflicts of Interest, data sharing, and preregistration during the preprint submission workflow for ingestion by preprint consumers.

COS has found that open science badges are an effective way to signal and promote open research practices (Kidwell et al., 2016, ttps://journals.plos.org/plosbiology/article?id=10.1371/journal.pbio.1002456"). With this award, we will extend the initial Sloan-supported work to a workflow for awarding badges for open practices based on author-assertions, and eventually, verified independently by the community. All content and tools will be open-source and developed to be integrated with other platforms, and badging will be machine-readable through the OSF API to enable interoperability with other services. To complete this work, we will engage the 26 OSF preprint communities and the others that collaborated with us for the Sloan work (e.g., eLife, ASAPbio, PLOS) to maximize potential for impact and use across services.

Open peer review integrations

Whatever its flaws, peer review is the most fundamental credibility indicator for scientific manuscripts. Making improvements so that authors of preprints hosted on OSF Preprint services can request a review from Peer Community In (PCl; https://peercommunityin.org/), PREreview (https://prereview.org/) or Hypothes.is (https://web.hypothes.is/) is a critical task in increasing the credibility of research shared via open services like preprints.

For a preprint submitted to $\mathrm{PCl}$, the editor may decide to recommend and write a recommendation that is published by the corresponding $\mathrm{PCl}$ along with all peer reviews that have been generated. Presently, $\mathrm{PCl}$ offers open review for preprints in Evolutionary Biology, Ecology, Paleontology, Animal Science, Entomology, Circuit Neuroscience, and Genomics. New $\mathrm{PCl}$ services are currently set up in Forest and Wood Sciences, Meta-Research, Prehistory, Mathematical and Computational Biology, and Registered reports; about 2,053 preprints presently hosted on OSF Preprint services are in these fields. At least one of our communities (AfricArXiv) is already partnering with $\mathrm{PCl}$ for strategic communication about community-driven peer review on the African continent, and we expect this integration will promote growth in both services.

We also seek to integrate with PREreview, a new, open-source, and free platform designed to engage the scientific community with collaborative and constructive peer review on preprints. Key features offered by the PREreview platform include the ability for researchers to sign in with their ORCID IDs, solicit preprint feedback, leave comments, and endorse others' reviews. 
Sustainability for developing world services

In 2018, COS initiated a transition of managing maintenance costs for OSF Preprints service to a shared-cost model for the 26 preprint services using the common infrastructure. For 2020 , total maintenance cost (DOls, hosting, storage, essential software maintenance for keeping operations effective and bug-free) is projected to be $\$ 229,225$ for publication of 33,650 preprints and postprints ( $\$ 6.81$ per paper). COS committed to fundraising $\$ 141,249(62 \%)$ and distributed the remaining costs $(\$ 87,976 ; 38 \%)$ by usage rates across the services. Some services are able to adapt to this distributed cost model easily, while groups operating a service for under-resourced communities in the developing world are more at risk. To give these groups time to develop support for their services during 2020 , we request $\$ 64,658$ to cover the total costs of six services, together accounting for 10,718 papers published in 2019: INA-rXiv $(\$ 48,989)$ for Indonesian research, arabixiv $(\$ 7,837)$ for Arabic languages, AfricArXiv $(\$ 1,958)$ for African research, IndiarXiv $(\$ 1,958)$ for Indian research, Frenxiv $(\$ 1,958)$ for French language research worldwide, and Agrixiv $(\$ 1,958)$ for agricultural research operated by an Indian open-access community.

\section{Milestones and Deliverables}

Add credibility signaling badges with link validation to OSF Preprints and make openly available for broader adoption

- Month 1: Sloan-funded project includes experimentally assessing effectiveness of Conflict of interest, Data sharing, and preregistration initial indicators $($ End date $=$ October 2020). We will analyze these data to inform planning for implementation priority and strategy.

- Month 2: Community outreach with OSF Preprint community servers, Badges committee and other stakeholders to share outcomes from Sloan study and gather feedback on badges for preprints.

- Month 3: Based on community and stakeholder engagement and analysis of user behavior, develop user personas, stories, and specifications for implementing interoperable, machine-readable badges on preprints.

- Months 4-9: Implement validated, machine-readable badges on preprints.

- Month 10: Develop and execute a communication and outreach strategy to promote preprint badges to community for encouraging usage and adoption.

- Months 11-12: Measure the usage and adoption by capturing the submission totals with each badge, views count totals for preprints with badges, and clicks on preprint badges to access the linked content.

Explore MVP integration of Open peer review

- Month 1: Engage the scholarly communications community to understand the needs for open review platforms (such as PCl and PREreview) to connect with OSF Preprints and establish the ideal workflows for integration of platforms.

- Month 2: Develop user personas, stories, and specifications for bi-directional integration between $\mathrm{PCl}$ and OSF Preprints infrastructure. 
- Month 3: Conduct technical analysis on open review platform integrations (e.g., $\mathrm{PCl}$ and PREreview) and explore the standards for transferring files and metadata to external platforms. Consult with external platforms that will be receiving preprints from OSF Preprints and other systems to ensure broad compatibility with as many platforms as possible with a standard schema.

- Month 4: Determine the technical approach for an MVP integration to transfer preprint file and metadata to other downstream platforms like open reviews, preservation, and formal peer review at journals.

- Months 5-11: Develop the transfer of preprint files and metadata. Test preprint file and metadata transfer with open review systems PCI and PREreview and others like Internet Archive for preservation.

- Month 12: Promote to community for encouraging usage and adoption

Sustainability for developing world preprint services

- Month 2: Develop fundraising strategy and outreach plan for all six developing world services. Include both individual service outreach, and collective outreach plan (i.e., opportunity for institutions to support individual service that fits their local priority, or all services to support the entire preprint ecosystem).

- Month 4: Each service completes outreach to four potential supporters, or secures funding for 2021+.

- Month 6: Each service completes outreach to eight potential supporters, or secures funding for 2021+.

- Month 9: Each service secures funding for 2021+.

- Month 11: Review and update sustainability plan based on revenues and long-term support commitments for individual services and for OSF Preprints community as a whole.

\section{Diversity, Equity, and Inclusion Statement}

Diversity and inclusion are organizational imperatives for COS. Our mission is to increase openness of research -- open means both increasing transparency/sharing and inclusivity/opportunity.

Our current staff of 44 (see http://cos.io/people/) includes 16 women and 28 men, with some diversity on other social identities. We encourage and actively recruit applications from members of groups underrepresented in science and technology.

We maintain the OSF as a free and open software service to maximize access and inclusion for researchers of any means and experience. For developers, we reduced the barrier to entry contributing with our easy to read and implement developer documentation and dockerization of the codebase for easy installation. The OSF code base has many contributions from a diverse group, including interns. 
As we further develop and improve the OSF infrastructure and interfaces, we prioritize WCAG AA compliance for accessibility and support translations of the pages in myriad languages. OSF offers diversity in file storage locations available to users: Frankfurt, Sydney, Montreal, and the U.S. are choices to meet user needs for institutional or governmental requirements.

COS has cultivated communities of practice in underserved areas to raise capacity of those research communities. For example, we include our preprint partners on this proposal from INA-rXiv (Indonesia), AfricArXiv (Africa), Indiarxiv (India), Frenxiv (French language, including developing world), Agrixiv (agricultural research operated by Indian partners), and Arabrxiv (Arabic languages). 